\title{
PROSPECTIVA E INTELIGENCIA ESTRATÉGICA APLICADA A LA MICRO, PEQUEÑA Y MEDIANA EMPRESA
}

\section{PROSPECTIVE AND BUSINESS STRATEGIC APPLIED TO MICRO, SMALL AND MEDIUM ENTERPRISES}

\section{INTELIGÊNCIA PROSPECTIVA E ESTRATÉGICA APLICADA ÀS MICRO, PEQUENAS E MÉDIAS EMPRESAS}

\author{
GUTARRA ROMERO_Roly Javier
}

Doctorando en Ciencias Sociales en El Colegio de Tamaulipas. Profesor-investigador en El Colegio de Tamaulipas. Email: roly.gutarra@gmail.com, México.

Recibido: 17 de mayo de 2019

Aprobado: 13 de julio de 2019

DOI: http: //dx.doi.org/10.22267/rtend.192001.110

\section{RESUMEN}

Las micro, pequeñas y medianas empresas (Mipymes) son la base de la estructura económica de un país. Su contribución al desarrollo económico y social es trascendental, pues generan empleo, aportan al Producto Bruto Interno (PBI), entre otros. Por tal motivo, es necesario que desarrollen procesos de gestión y toma de decisiones innovadoras para hacer frente al entorno de incertidumbre, complejidad y competitividad en el que se encuentran inmersas.

La investigación consistió en utilizar la prospectiva e inteligencia estratégica para proponer una metodología que facilite la toma de decisiones de las Mipymes en el mediano y largo plazo. El resultado fue una metodología estructurada en tres fases: Conociendo la empresa, Posibilidades de la empresa y Estrategias para la empresa. No obstante, ésta deberá ser aplicada en diversas Mipymes para validarla y generar información respecto a la planeación y proceso de toma de decisiones. 
Palabras clave: mipymes, prospectiva, inteligencia, estrategia JEL: M10, M15, M19

\begin{abstract}
The micro, small and medium enterprises (MSMEs) are the basis of the economic structure of a country. Its contribution to economic and social development is transcendental; since they generate employment, contribute to the Gross Domestic Product (GDP), among others.
\end{abstract}

For this reason, it is necessary that they develop innovative management and decision-making processes to face the environment of uncertainty, complexity and competitiveness in which they are immersed.

The research consisted of using foresight and strategic intelligence to propose a methodology that facilitates the decision making of MSMEs in the medium and long term. The result was a structured methodology in three phases: Knowing the company, Possibilities of the company and Strategies for the company. However, this must be applied in various MSMEs to validate it and generate information regarding the planning and decision-making process.

Keywords: MSMEs, prospective, intelligence, strategy

JEL: M10, M15, M19

\title{
RESUMO
}

As micro, pequenas e médias empresas (MPMEs) são a base da estrutura econômica de um país. Sua contribuição para o desenvolvimento econômico e social é transcendental, pois geram emprego, contribuem para o Produto Interno Bruto (PIB), entre outros.

Por essa razão, é necessário que desenvolvam processos inovadores de gestão e tomada de decisão para enfrentar o ambiente de incerteza, complexidade e competitividade em que estão imersos.

A pesquisa consistiu no uso de prospectiva e inteligência estratégica para propor uma metodologia que facilite a tomada de decisão das MPMEs a médio e longo 
prazo. O resultado foi uma metodologia estruturada em três fases: Conhecendo a empresa, Possibilidades da empresa e Estratégias para a empresa. No entanto, isso deve ser aplicado em várias MPMEs para validá-lo e gerar informações sobre o processo de planejamento e tomada de decisões.

Palavras-chave: MPMEs, prospectiva, inteligência, estratégia JEL: M10, M15, M19

\section{INTRODUCCIÓN}

Las micro, pequeñas y medianas empresas (Mipymes) constituyen la base de la estructura económica y empresarial de un país, puesto que representan, aproximadamente, el 99\% del total de empresas, verbigracia en Latinoamérica representan el 99.5\% ${ }^{1}$ y en la Unión Europea representan el $99.8 \%{ }^{2}$. Estos porcentajes demuestran la importancia de dichas unidades económicas en un país, pues, además, de generar empleo, contribuyen al desarrollo productivo, económico y social.

Sin embargo, el actual contexto globalizado las obliga a ser más competitivas para seguir en el mercado, y al mismo tiempo, logren insertarse a cadenas de valor nacionales e internacionales. Por tal motivo, las Mipymes necesitan desarrollar procesos de gestión y de toma de decisiones anticipadas con el fin de prever los cambios sociales y tecnológicos, el ingreso de nuevos competidores y nuevos productos, cambios en el mercado, entre otros; y así, hacer frente a un futuro complejo e incierto.

Para tal efecto, las Mipymes deben realizar procesos de planeación de mediano y largo plazo, considerando el entorno externo e interno de la organización, las tendencias emergentes, los actores preponderantes, nuevos mercados y productos, etc. Por lo tanto, el autor propone el uso de herramientas prospectivas y de inteligencia estratégica para transformar la información en conocimiento operable y así facilitar la toma de decisiones en este grupo de empresas.

1 Las Mipymes en países como Perú, México, Colombia, Ecuador, entre otros., representan el 99\% de su estructura empresarial, según datos obtenidos de la CEPAL. Para más información revisar en: https://www. cepal.org/sites/default/files/events/files/nicolo_giglo.pdf

2 La Unión Europea conceptualizan a las micro, pequeñas y medianas empresas como "Pymes" y representan el 99\% del total de empresas. Para obtener más información revisar en: https://europa.eu/european-union/topics/enterprise_es 
Esta investigación tiene como propósito ofrecer una metodología para mejorar el proceso de planeación y toma de decisiones de las Mipymes, la cual deberá ser aplicada en diversas Mipymes para validarla y obtener resultados de corte cuantitativo y cualitativo.

El artículo se estructuró en tres secciones. La primera consistió en contextualizar a las Mipymes, para luego dar a conocer el porcentaje aproximado de dichas organizaciones en América Latina y la Unión Europea, también, se buscó explicar el entorno global en el cual se encuentran inmersas y sus debilidades frente a ese proceso. En la segunda se abordaron los conceptos de prospectiva e inteligencia estratégica y cómo estas se pueden usar en la gestión de las Mipymes. En la tercera se explicó la metodología que vincula la prospectiva e inteligencia en el ámbito empresarial y como ambas se complementan para generar estrategias sistémicas y holísticas de mediano y largo plazo.

\section{CONTEXTO Y CARACTERIZACIÓN DE LAS MIPYMES}

La estructura empresarial de un país está compuesta en su gran mayoría por Mipymes, tal como se aprecia en la Figura 1, donde se muestra el porcentaje de Mipymes en América Latina y la Unión Europa.
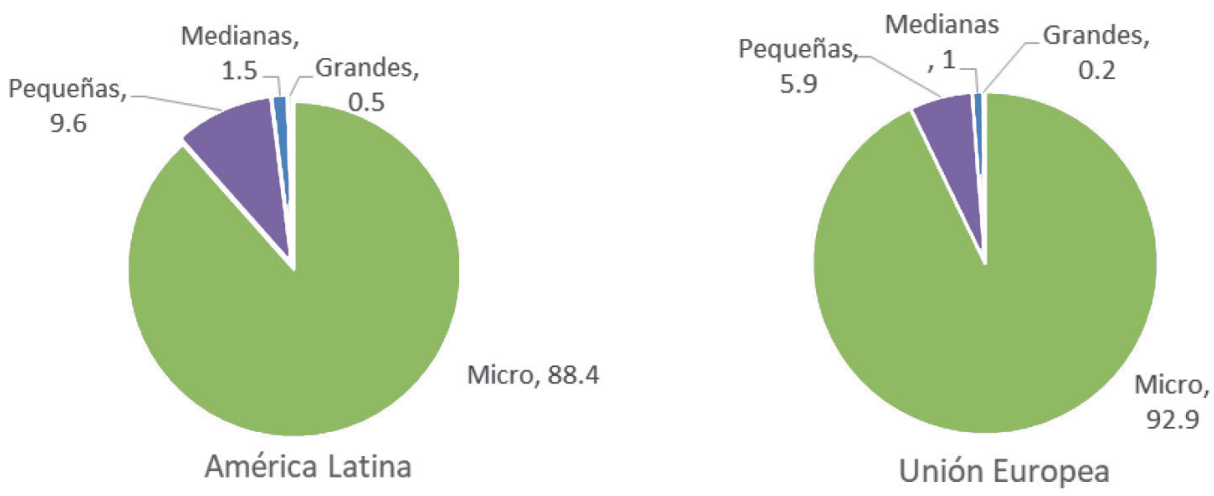

\section{Figura 1}

Porcentaje de Mipymes en América Latina y la Unión Europea - 2016

Fuente: Elaboración propia con información de Dini y Stumpo, 2018. 
Es importante precisar que no existe un consenso entre el sector público, privado y académico para definir y clasificar a estas unidades económicas, por tal motivo, se toma como ejes de definición al número de trabajadores y la facturación anual. No obstante, en algunos países, además del número de trabajadores y facturación anual, se consideran otros parámetros de medición como el de sectores económicos, lo cual dificulta las comparaciones entre Mipymes de diferentes países.

Por tal motivo, la Organización Mundial del Comercio en su informe sobre el comercio mundial $2016^{3}$ uniformiza la caracterización de las Mipymes y utiliza el examen de los indicadores nacionales sobre Mipymes de la Corporación Financiera Internacional ${ }^{4}$ disponibles para 132 economías de diferentes niveles de desarrollo económico y correspondientes principalmente a los años 2007 y 2008 , en la cual afirma que un gran número de países utilizan las siguientes clasificaciones: microempresas tienen como 10 empleados como máximo, pequeñas empresas tienen entre 10 y 50 empleados, y las medianas empresas tienen entre 50 y 250 empleados (2016: 17).

Otro rasgo importante es que de acuerdo con la Tabla 1, del conjunto total de Mipymes de los países que forman parte de la Organización Mundial del Comercio $^{5}$, en los países desarrollados las microempresas representan el 87.1 $\%$, las pequeñas el 10.7 y las medianas el $2.2 \%$; en los países en desarrollo las microempresas representan el $80.5 \%$, las pequeñas el 15.6 y las medianas el $3.9 \%$. También, en dicha tabla se toma en consideración a los países miembros del foro G-20, otros países en desarrollo y los países menos adelantados. Los datos obtenidos revelan la existencia de un alto porcentaje de microempresas en un país, pero muchas de estas son endebles por problemas de gestión, capacitación, financiamiento, entre otros, aumentando sus probabilidades de fracasar y salir del mercado (Dini y Stumpo, 2018).

3 Informe sobre el Comercio Mundial 2016 - Igualdad de condiciones para el comercio de Pymes.

4 La Corporación Financiera Internacional entidad del Grupo Banco Mundial, es la principal institución internacional de desarrollo dedicada exclusivamente al sector privado en los países en desarrollo.

5 Para obtener más información sobre los países miembros de la Organización Mundial del Comercio y su clasificación en países desarrollados, en desarrollo y menos adelantados revisar en: https://www.wto.org 


\section{Tabla 1}

Porcentaje de micro, pequeñas y medianas empresas en el conjunto total de Mipymes de los países pertenecientes a la Organización Mundial del Comercio.

\begin{tabular}{|l|l|l|l|}
\cline { 2 - 4 } \multicolumn{1}{c|}{} & $\begin{array}{l}\text { \% de } \\
\text { microempresas }\end{array}$ & $\begin{array}{l}\text { \% de pequeñas } \\
\text { empresas }\end{array}$ & $\begin{array}{l}\text { \% demedianas } \\
\text { empresas }\end{array}$ \\
\hline Países desarrollados & 87,1 & 10,7 & 2,2 \\
\hline Países en desarrollo & 80,5 & 15,6 & 3,9 \\
\hline $\begin{array}{l}\text { Países en desarrollo del } \\
\text { G-20 }\end{array}$ & 82,1 & 13,2 & 4,7 \\
\hline Otros países en desarrollo & 80,5 & 14,9 & 4,5 \\
\hline PMA & 78,6 & 20,7 & 0,6 \\
\hline Total & 82,9 & 13,8 & 3,3 \\
\hline
\end{tabular}

Fuente: Elaboración propia con datos del Informe la Organización Mundial del Comercio (2016).

Con relación a las actividades económicas que realizan las Mipymes, es importante señalar que éstas por naturaleza son heterogéneas; es decir, se encuentran inmersas en el sector industrial, comercial, de servicios y pueden ser desde pequeños proveedores de servicios tradicionales hasta proveedores de servicios altamente calificados, bienes tecnológicos, entre otros. La diferencia de calidad entre los bienes y/o servicios producidos por dichas unidades económicas se debe a que la mayoría no cuenta con las competencias o capacidades técnicas necesarias como financiamiento, innovación, capacitación, asociatividad, gestión, planeación, entre otras (Correa, Leiva y Stumpo, 2018), lo cual dificulta su inserción a cadenas de valor nacionales e internacionales y consecuentemente sus procesos de exportación.

Si bien es cierto, las falencias señaladas en el párrafo anterior tienen muchas aristas, en algunos casos se deben al incorrecto diseño y/o aplicación de las políticas públicas, pues estas abordan el acceso a financiamiento, tecnologías, capacitación, practicas asociativas, etc., sin tener un objetivo en común o coherencia entre los objetivos, programas y presupuestos. También, influye la limitada trayectoria de aprendizaje de las instituciones involucradas en la ejecución e implementación de las políticas (Goldstein y Kulfas, 2011, Dini y Rueda, 2018). 
Además, las debilidades de las Mipymes se deben a que, muchas de estas, no poseen una cultura de la planeación a mediano y largo plazo, imposibilitando con esto una mejor toma de decisiones de cara hacia al futuro. Aunado a lo anterior, los propietarios de Mipymes no conciben a éstas como una unidad compleja, pues administran la empresa desde una perspectiva analítica y reduccionista, es decir, dividen a la empresa en diversas áreas (legal, recursos humanos, ventas, logística, etc.) y gestionan tales áreas en forma independiente (Lacayo y García, s.f.), sin comprender que la empresa es un todo, un ente sistémico y complejo ${ }^{6}$, en la cual interactúan sus elementos entre sí para alcanzar un objetivo.

Por lo anterior, la generación de planes estratégicos de mediano y largo plazo utilizando la prospectiva e inteligencia estratégica, serán trascendentales para que estas empresas logren trascender en el tiempo, debido a que, el entorno actual en el que se encuentran inmersas es muy competitivo y el futuro que les depara es cada vez más complejo, en donde las empresas ya no compiten a nivel local, sino a nivel internacional y donde las nuevas oportunidades de negocios estarán ligadas a una alta capacidad en el uso de tecnologías. Es decir, el mercado demandará Mipymes competitivas, innovadoras, tecnológicas y de alta eficiencia productiva, que desarrollen productos y presten servicios de calidad con un grado alto o medio de intensidad tecnológica.

El contexto actual y el futuro que les depara a las Mipymes las obliga a anticiparse al futuro y mejorar su toma de decisiones para construir el mejor futuro dentro de los futuros posibles y no desaparecer del mercado. Por lo tanto, el uso de la prospectiva e inteligencia estratégica son necesarios para lograr su desarrollo, pues permitirá realizar diagnósticos del contexto interno y externo de la empresa, identificar actores y variables clave, analizar tendencias, generar escenarios futuros y diseñar estrategias para construir un futuro deseado.

La importancia de buscar la trascendencia en el tiempo de las Mipymes radica en el hecho de que tienen la capacidad de impulsar el desarrollo económico de un país y colaborar para una mejor distribución de ingresos. Al respecto Goldstein y Kulfas (2011) afirman lo siguiente:

6 Según la Real Academia de la Lengua Española el concepto complejo se refiere al conjunto o unión de dos o más cosas que constituyen una unidad. 
Las pymes pueden ayudar a la articulación de las cadenas productivas [...] apostando a mejorar el contenido tecnológico y a un uso más intensivo del conocimiento en la producción [...] En suma, las pymes pueden aportar a mejorar la competitividad de la economía; al desarrollo productivo, sectorial y regional, y al mejoramiento de las condiciones sociolaborales y la distribución del ingreso (p. 475).

Finalmente, la importancia de estas unidades económicas se fundamenta en el hecho de que si son adecuadamente gestionadas y cuentan con herramientas de planeación a mediano y largo plazo, así como con herramientas para una toma de decisiones estratégica y anticipada, pueden convertirse en empresas de mayor tamaño, con bases sólidas y más competitivas en un mercado nacional e internacional.

\section{PROSPECTIVA E INTELIGENCIA ESTRATÉGICA}

Cuando se habla del futuro desde un enfoque prospectivo se hace referencia a múltiples futuros y no solo a un futuro determinado, es decir, queda en la voluntad del ser humano u organización construir el futuro que desee, elegido éste de un conjunto de futuros posibles. Puesto que, para la prospectiva el futuro es susceptible de ser creado con base a requisitos mínimos (libertad, voluntad y poder) y la aplicación de metodologías que engloban herramientas de corte cualitativo y cuantitativo.

Para lograr un futuro deseado, es esencial que el tomador de decisiones se anticipe al futuro, a fin de tener una mayor claridad al momento de diseñar las estrategias y acciones que construirán el futuro. Al respecto Godet menciona que "La anticipación no tiene mayor sentido si no es que sirve para esclarecer la acción" (2007: 6). Ese el motivo por el cual se genera el vínculo indisociable entre la prospectiva y la estrategia, surgiendo el concepto de prospectiva estratégica (Godet, 2007).

Efectivamente, la prospectiva y estrategia son elementos complementarios e indisociables, así lo señala Gaston Berger:

¿Cómo podríamos pensar en actuar como un estratega sin "mirar a lo lejos, a lo ancho, a lo profundo: tomar riesgos, pensar en el hombre" tal como lo hace la prospectiva [...] ¡Por supuesto sería imposible! Y, en sentido inverso, [...] "contemplando el futuro se transforma el presente"; de este modo la anticipación invita a la acción. (citado en Godet, 2007: 9). 
En ese sentido, la prospectiva estratégica tiene diversas conceptualizaciones, entre ellas -y la que se utilizará como referencia para la presente investigaciónse encuentra la de Miklos y Arroyo (2011: 126) los cuales señalan que:

Así pues, la prospectiva es un método para anticiparse al futuro. También es una herramienta para innovar y cambiar. Su función central es la de prevenir a partir de lo que ha pasado (retrospectiva) y que se expresa en el presente (coyuntura). Pero no es solamente una proyección de las tendencias del pasado, sino la construcción de otras posibilidades (visiones) que oscilan entre la catástrofe (el peor lugar adonde arribar) y la utopía (el mejor lugar adonde llegar), proponiendo estratégicamente el mejor lugar deseable-posible (futurible), lo cual implica ir más allá del presente y su tendencia.

Por otra parte, al considerar el entorno actual en el que se encuentran inmersas las Mipymes, que es de globalización, competitividad, desarrollo tecnológico, nuevos mercados, entre otras, hace imprescindible el uso de la prospectiva en sus procesos de gestión, para que así puedan hacer frente a tales retos y logren construir el mejor futuro posible.

La aplicación de la prospectiva en al ámbito empresarial explora de manera reflexiva los posibles escenarios futuros de una empresa y genera estrategias para anticiparse a los mismos. Sobre el particular, Medina (s.f.: 8) conceptualiza la prospectiva desde el punto de vista empresarial

Como un modelo de análisis organizacional que incluye el análisis de los entornos globales con el fin de identificar hechos portadores de futuro, fuerzas motoras de cambio, tendencias emergentes que conlleven a decisiones sobre incursión en nuevos mercados, reorientación de productos y servicios, entre otras.

La importancia de la prospectiva aplicada a las Mipymes se da por el ambiente de incertidumbre y complejidad que tienen éstas de cara al futuro, así también, por la visión de corto plazo y reduccionista de los propietarios y/o gestores de aquellas, propiciando que la prospectiva empresarial se convierta junto a la inteligencia empresarial en herramientas clave para anticipar el futuro, facilitar la toma de decisiones y desarrollar procesos de innovación. Ciertamente, se afirma que: "La prospectiva se ha convertido en una herramienta de competitividad para las empresas, una manera de facilitar la toma de decisiones directivas" (Serra del Pino y Herrera, 2017). 
Además, el uso de la prospectiva en las Mipymes hará que divisen nuevas oportunidades de negocios; se adelanten en el uso y aplicación de nuevas tecnologías; que identifiquen tendencias históricas, emergentes y dominantes; que incorporen el pensamiento sistémico, holístico y de largo plazo en la toma de decisiones; y que generen una cultura de planeación prospectiva. Todo lo anterior con el objetivo de reducir la incertidumbre del futuro y transformarla en riesgo ${ }^{7}$.

Sin embargo, el uso de la prospectiva por parte de las Mipymes es limitado, contrario sensu ocurre con las grandes empresas como, por ejemplo, Royal Dutsh Shell, Daimler-Chrysler, Volvo, Philips, etc., pues éstas la aplican hace unas décadas para la elaboración de sus planes estratégicos y generar estrategias sistémicas de mediano y largo plazo. El restringido uso de la prospectiva en las Mipymes se debe, entre otras cosas, al desconocimiento de la existencia de la herramienta por parte de los propietarios y gestores, creencia infundada de altos costos de implementación, no poseer recurso humano capacitado, etc.

Por otro lado, con la inteligencia estratégica se busca transformar la información existente en el entorno y convertirla en conocimiento, a través de un proceso de recopilación, procesamiento y análisis de la información existente en el entorno externo e interno. (Shulsky y Schmitt, 2002/1991). Al respecto el Comité $\mathrm{Church}^{8}$ señala que "La inteligencia es el producto resultante de la recolección, comparación, evaluación, análisis, integración e interpretación de toda la información recolectada" (citado por Troy, 1992: 9).

Así, el proceso de transformar la información en conocimiento se desarrolla en forma cíclica, lo que se conoce como el "Ciclo de la Inteligencia", ésta según Navarro (2004) consiste en seguir un conjunto de etapas concatenadas, que comienza con la planificación y dirección, obtención de la información, proceso, análisis y generación de inteligencia, y difusión.

Las particularidades de la inteligencia estratégica la convierten en una herramienta valiosa y complementaria a la prospectiva, puesto que, el vínculo entre ambas

\footnotetext{
7 Es importante convertir la incertidumbre del futuro en riesgo, porque el riesgo si es capaz de controlarse y tomar las acciones necesarias para evitar impactos negativos en las empresas.

8 El comité Church es la manera informal como se le denomina al Comité Selecto del Senado de los Estados Unidos para el Estudio de las Operaciones Gubernamentales Respecto a las Actividades de Inteligencia.
} 
permitirá que se generen estrategias de corto, mediano y largo plazo de forma holística y sistémica para que los propietarios y/o gestores de Mipymes tomen mejores decisiones.

Es necesario precisar que se han realizado estudios donde se analiza la pertinencia de la inteligencia estratégica en la gestión de organizaciones ${ }^{9}$, así según Aguirre (2015:103) la inteligencia estratégica ${ }^{10}$ es “...una herramienta clave para dirigir compañías o para proponer planes estratégicos de una organización...”. Asimismo, Cabrera (2015: 193) señala que la inteligencia estratégica es “... una herramienta prioritaria para la toma de decisiones, tomando como directa consecuencia una considerable reducción de la situación de incertidumbre". Ambos conceptos demuestran la importancia de la inteligencia estratégica en la gestión y toma de decisiones de las organizaciones, cabe precisar que una de las vertientes de la inteligencia estratégica es la inteligencia empresarial, al respecto Cubillo señala lo siguiente:

La inteligencia empresarial, designa [...] aquel conjunto de capacidades propias o movilizables por una entidad lucrativa, destinadas a asegurar el acceso, capturar e interpretar y preparar conocimiento e información con alto valor agregado para apoyar la toma de decisiones requeridas por el diseño y ejecución de su estrategia competitiva. (1997: 261).

El uso de la prospectiva e inteligencia estratégica en la gestión de las Mipymes permitirá que éstas innoven procesos y productos, identifiquen futuras amenazas y oportunidades, enfrenten nuevas formas de competencia, identifiquen tendencias sociales y tecnológicas, se inserten a cadenas de valor internacionales, realicen monitoreos de sus competidores, etc. Estas acciones serán decisivas para que dichas organizaciones económicas puedan trascender en el tiempo y ser competitivas en un mundo cada vez más complejo e incierto. Por tal motivo, con relación a las organizaciones económicas Godet señala lo siguiente:

Las amenazas y oportunidades provenientes del entorno general, nos muestra que no se puede limitar, en nombre del beneficio a corto plazo, sólo al análisis del entorno

\footnotetext{
9 Entiéndase por organizaciones al conjunto de empresas públicas y privadas, dentro de las cuales se encuentran inmersas las Mipymes.

10 Se hace referencia al término inteligencia estratégica porque es la base conceptual y metodológica de la inteligencia empresarial. En efecto, la inteligencia empresarial es la inteligencia estratégica aplicado al mundo empresarial.
} 
competitivo como podríamos deducir de la lectura de las primeras obras de Michael Porter. Las múltiples incertidumbres, que sobre todo pesan a largo plazo en el contexto general, nos muestran el interés de la construcción de escenarios globales para esclarecer la elección de las opciones estratégicas y asegurar la perennidad del desarrollo (2007: 8).

Asimismo, es importante que los propietarios y/o gestores de las Mipymes cambien sus modelos estructurales de toma de decisiones de corto plazo, y al mismo tiempo, eviten enfocarse en producir bienes y bridar servicios solo para su entorno local y, por el contrario, busquen insertarse a cadenas de producción internacionales y aprovechar las oportunidades que ofrecen los mercados internacionales.

\section{METODOLOGÍA PARA DESARROLLAR LA PROSPECTIVA E INTELIGENCIA ESTRATÉGICA EN LAS MIPYMES}

Para que las Mipymes puedan utilizar la prospectiva e inteligencia estratégica en sus procesos de planeación y toma de decisiones, es necesario que cuenten con un proceso metodológico que englobe fases y herramientas esenciales que conlleven a la generación de estrategias de base sistémica, holística de mediano y largo plazo.

Dicho proceso metodológico tiene que adecuarse a las características y necesidades de las Mipymes, al mismo tiempo, debe ser de fácil uso y aplicación por parte de los tomadores de decisiones y colaboradores de la empresa encargados del proceso de planeación. También, es importante que el uso de la prospectiva e inteligencia estratégica no impliquen gastos excesivos y tampoco generen la necesidad de desarrollar infraestructuras $\operatorname{costosas}^{11}$; por el contrario, se busca que sean herramientas que coadyuven al ahorro económico e incrementen las utilidades de la empresa.

Hay que mencionar, además, que una de las características de las Mipymes es su fácil adaptabilidad a las circunstancias y necesidades, por ende, son organizaciones en las que se puede desarrollar con mayor practicidad procesos de innovación en la gestión. (Dini y Stumpo, 2018). Consecuentemente, es más

11 El desarrollo de infraestructuras implica: la implementación de plataformas virtuales, la compra de softwares de procesamiento y análisis de datos, creación de un área específica de investigación prospectiva, entre otras. 
factible la aplicación de la prospectiva e inteligencia empresarial en este tipo de empresas.

Dicha característica se debe a que la propiedad y/o gestión de la empresa se encuentra en poder de un reducido grupo de personas, y en algunos casos, la propiedad y gestión se encuentran en manos de una sola persona (dueñoempresario). Esto genera un rápido proceso de toma de decisiones, debido a que, el poder dentro de la organización es lineal y ostentado por muy pocas personas o solo por el propietario, contrario sensu ocurre en las grandes empresas, este rasgo distintivo de las Mipymes facilita la implementación de nuevos procesos, herramientas y metodologías de gestión y administración dentro de la organización.

La metodología presentada en este documento tiene por finalidad ofrecer a los propietarios y/o gestores de las Mipymes una opción distinta para realizar sus procesos de planeación de mediano y largo plazo, a través del análisis interno y externo de la organización, identificación de tendencias emergentes y dominantes de corte social y tecnológico, identificación de variables clave; a fin de, generar estrategias para la construcción del futuro deseado de la empresa.

Como se puede observar en la Figura 2 el proceso metodológico está constituido por tres fases prospectivas concatenadas: Conociendo la empresa, Posibilidades de la empresa y Estrategias para la empresa. Estas tres etapas se encuentran enmarcadas dentro del proceso de inteligencia empresarial: Planeación, Investigación y recolección, Análisis y producción, y Diseminación y explotación. Constituyéndose así una metodología integral, dinámica y cíclica, ya que, una vez culminada la última etapa se vuelve a iniciar con la primera para retroalimentarla; esto debido a que, el mundo se encuentra en constante cambio y por lo tanto es imprescindible que continuamente se realicen monitoreos del cumplimiento de los objetivo y estrategias, con la finalidad de reformularlas, si es necesario, para alcanzar el futuro deseado.

Es importante precisar que, existe una relación directa y complementaria entre las fases de la prospectiva y la inteligencia. Es así como, la primera fase de la prospectiva empresarial Conociendo la empresa se vincula con la Planeación e Investigación y recolección (primera y segunda etapa de la inteligencia 
empresarial); seguidamente, la segunda fase Posibilidades de la empresa se vincula con la tercera etapa Análisis y producción; finalmente, la tercera fase Estrategias para la empresa se vincula con cuarta etapa Diseminación y explotación.

Ahora bien, en cada fase prospectiva (conociendo la empresa, posibilidades de la empresa y estrategias para la empresa) se utilizan diversas herramientas para la obtención de productos prospectivos clave, las cuales servirán de insumo para el desarrollo de las otras fases, además, pueden ser aprovechadas por las distintas áreas de la organización de acuerdo con sus intereses.

Precisamente, para un mejor entendimiento de la metodología prospectiva se procederá a explicar el proceso de cada una de las fases y los vínculos de éstas con las etapas de la inteligencia empresarial. Al mismo tiempo, se sugerirán las herramientas a utilizar para el cumplimiento de cada fase.

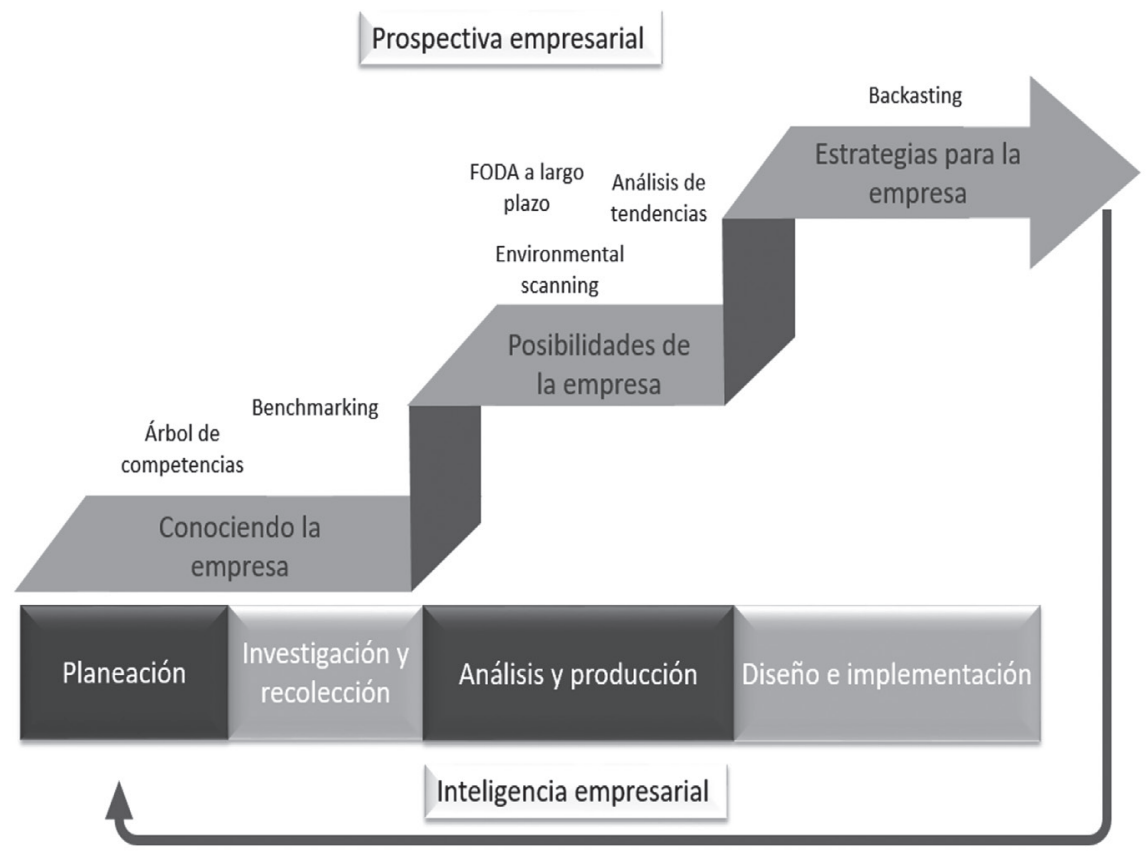

\section{Figura 2}

Proceso metodológico de prospectiva e inteligencia empresarial para las Mipymes Fuente: Elaboración propia. 


\section{- Primera fase - Conociendo la empresa. -}

En esta fase se da el primer acercamiento integral para conocer a la empresa, con la finalidad de averiguar el sector de negocios en el cual desarrolla sus actividades, conocer sus procesos, los productos y/o servicios que ofrecen, el recurso humano con el que cuenta, identificar competidores, entre otros.

Para lograr esta fase prospectiva se hace una vinculación con las dos primeras etapas de la inteligencia empresarial: 'Planeación e Investigación y recolección', debido a que en la etapa de planeación se definen los objetivos del estudio prospectivo, se identifican los temas críticos de la organización, la agenda a desarrollar, las personas que la llevarán a cabo y los recursos económicos a utilizarse; y en la etapa de investigación y recolección se delimita el plan de búsqueda de la información, las fuentes y redes de información a utilizarse, se definen las técnicas de investigación y las herramientas a emplear.

Para esta fase se recomienda utilizar las siguientes herramientas: Árbol de competencias y Benchmarking. Es necesario precisar que, ambas herramientas sirven para conocer el entorno interno y externo de la empresa, así como para visualizar a los competidores (Ver Tabla 2).

\section{Tabla 2}

Herramientas utilizadas para desarrollar la fase Conociendo la empresa

\begin{tabular}{|c|c|c|}
\hline Herramienta & Ventajas & Limitaciones \\
\hline $\begin{array}{l}\text { Árbol de competencias } \\
\text { Busca conocer } \\
\text { integralmente } \\
\text { a las Mipymes } \\
\text { para identificar } \\
\text { sus competencias } \\
\text { distintivas y su } \\
\text { dinámica }\end{array}$ & $\begin{array}{l}\checkmark \text { Visión integral } \\
\checkmark \text { Identificación del Know } \\
\text { how de la empresa } \\
\checkmark \text { Identificación de competen- } \\
\text { cias técnicas de la empresa } \\
\text { (gestión, económico, tecno- } \\
\text { lógicos, normativos, socia- } \\
\text { les, políticos, etc.) } \\
\checkmark \text { Identificación de líneas de } \\
\text { productos y servicios }\end{array}$ & $\begin{array}{l}\checkmark \text { Se limita al diagnóstico } \\
\text { interno de la } \\
\text { organización. } \\
\checkmark \text { Diagnóstico del } \\
\text { pasado y presente de la } \\
\text { organización. }\end{array}$ \\
\hline
\end{tabular}




\begin{tabular}{|c|c|c|}
\hline $\begin{array}{l}\text { Benchmarking } \\
\text { Proceso continuo de } \\
\text { comparar productos, } \\
\text { servicios y prácticas } \\
\text { de los competidores } \\
\text { o de las compañías } \\
\text { líderes en el sector } \\
\text { de negocios, para la } \\
\text { mejora continua de la } \\
\text { organización. }\end{array}$ & $\begin{array}{l}\checkmark \text { Proceso continuo y siste- } \\
\text { mático } \\
\checkmark \text { Comprender el funciona- } \\
\text { miento de la organización } \\
\checkmark \text { Conocer los procesos, pro- } \\
\text { ductos y servicios de los } \\
\text { competidores } \\
\checkmark \text { Identifica oportunidades de } \\
\text { mejora } \\
\checkmark \text { Ayuda a formular planes de } \\
\text { acción }\end{array}$ & $\begin{array}{l}\checkmark \text { Confundir en medir cos- } \\
\text { tos financieros cuando } \\
\text { se debe medir el valor } \\
\text { de la organización } \\
\checkmark \\
\checkmark \text { Es necesario contar con } \\
\text { recurso humano capa- } \\
\text { citado } \\
\checkmark \\
\checkmark \text { Dificultad para acceder } \\
\text { a información primaria } \\
\text { y secundaria }\end{array}$ \\
\hline
\end{tabular}

Fuente: Elaboración propia con información obtenida de Gándara, Muñoz y Luna (2014) para la realización del Árbol de problemas; y de Intxaurburu y Ochoa (2005) para la realización del Bechmarking.

\section{- Segunda fase - Posibilidades de la empresa. -}

En esta fase se busca explorar los escenarios futuros de la Mipymes de cara al futuro a nivel de nuevos mercados, productos, competidores, tecnología, etc. Es así como, para la consecución de las Posibilidades de la empresa se hace una vinculación con la segunda etapa de la inteligencia estratégica, 'Análisis y producción', debido a que en esta etapa se realiza el procesamiento de la información obtenida en la fase Conociendo la empresa, es decir, se transforma la información existente en conocimiento operable, además, se utilizan otras herramientas para obtener información del entorno externo de la empresa, identificar tendencias y generar escenarios futuros.

Consecuentemente, se recomiendan las siguientes herramientas: Environmental scanning, Análisis de tendencias y FODA a largo plazo (Ver tabla 3). La primera herramienta sirve para realizar una exploración del entorno de la organización, la segunda sirve para identificar tendencias emergentes y dominantes de corte social y tecnológica que pueden afectar a la empresa, y la última sirve para generar escenarios futuros sobre los posibles comportamientos de la empresa. 
Tabla 3.

Herramientas utilizadas para desarrollar la fase Posibilidades de la empresa

\begin{tabular}{|c|c|c|}
\hline Herramientas & Ventajas & Limitaciones \\
\hline $\begin{array}{l}\text { Environmental scanning } \\
\text { Tiene como propósito } \\
\text { explorar sistemáticamente } \\
\text { el entorno externo } \\
\text { de las Mipymes para } \\
\text { comprender el ritmo de } \\
\text { cambio de su entorno e } \\
\text { identificar oportunidades } \\
\text { y desafíos futuros }\end{array}$ & $\begin{array}{l}\checkmark \text { Análisis desde la pers- } \\
\text { pectiva económica, } \\
\text { social, ambiental, cul- } \\
\text { tural, política, tecnoló- } \\
\text { gica, entre otras. } \\
\checkmark \text { Proporcionar bases } \\
\text { para el desarrollo de } \\
\text { nuevas inversiones } \\
\checkmark \text { Identificar los cambios } \\
\text { anticipadamente }\end{array}$ & $\begin{array}{l}\checkmark \text { Se limita al diagnósti- } \\
\text { co externo de la orga- } \\
\text { nización } \\
\checkmark \text { Dificultad para acce- } \\
\text { der a información } \\
\checkmark \text { Necesidad de contar } \\
\text { con recurso humano } \\
\text { capacitado }\end{array}$ \\
\hline $\begin{array}{l}\text { Análisis de tendencias } \\
\text { Proceso continuo } \\
\text { de identificación de } \\
\text { tendencias emergentes y } \\
\text { dominantes que influirán } \\
\text { en el futuro de las } \\
\text { Mipymes }\end{array}$ & $\begin{array}{l}\checkmark \text { Identificar variables } \\
\text { cuyo impacto es de } \\
\text { largo plazo } \\
\checkmark \text { Identificar tendencias } \\
\text { sociales y tecnológicas } \\
\checkmark \text { Prever las consecuen- } \\
\text { cias de sus impactos }\end{array}$ & $\begin{array}{l}\checkmark \text { No identifica variables } \\
\text { de corto y mediano } \\
\text { plazo } \\
\checkmark \text { Necesidad de contar } \\
\text { con recurso humano } \\
\text { capacitado } \\
\checkmark \text { Dificultad para acce- } \\
\text { der a información pri- } \\
\text { maria y secundaria }\end{array}$ \\
\hline $\begin{array}{l}\text { FODA a largo plazo } \\
\text { Facilita la obtención de } \\
\text { conclusiones sobre la } \\
\text { forma como las Mipymes } \\
\text { serán capaces de afrontar } \\
\text { los cambios futuros en el } \\
\text { entorno externo e interno } \\
\text { de la organización }\end{array}$ & $\begin{array}{l}\checkmark \text { Enfrentar y anticiparse } \\
\text { a los cambios futuros } \\
\checkmark \text { Proceso de largo plazo } \\
\text { y sistémico } \\
\checkmark \text { Generación de esce- } \\
\text { narios a partir de los } \\
\text { cuatro ejes }\end{array}$ & $\begin{array}{l}\checkmark \text { No identifica variables } \\
\text { de corto y mediano } \\
\text { plazo } \\
\checkmark \text { Necesidad de contar } \\
\text { con recurso humano } \\
\text { capacitado }\end{array}$ \\
\hline
\end{tabular}

Fuente: Elaboración propia con información obtenida de Jackson (2013) para la realización del Environmental scanning; de Ortega (2014) para la realización del Análisis de tendencias; y del Ministerio de Planificación Nacional y Política Económica (2015) para la realización FODA a largo plazo. 


\section{- Tercera fase - Estrategias para la empresa. -}

En esta última fase prospectiva se diseñarán las estrategias que servirán para construir el escenario apuesta ${ }^{12}$ de la Mipyme. Consiguientemente, para el desarrollo de este proceso se hace una vinculación con la tercera etapa de la inteligencia estratégica, 'Diseminación y explotación'; puesto que, en esta etapa se utiliza la información obtenida en la fase Posibilidades de la empresa con la finalidad de generar productos -conocimiento operable- en forma oportuna y pertinente para el uso por parte del tomador de decisiones (propietarios y/o gestores de Mipymes).

En efecto, el uso de los productos de inteligencia se realizará a través del diseño de estrategias, las cuales se irán monitoreando para verificar si logran construir el escenario apuesta. Con el objetivo de realizar dicho proceso se recomienda utilizar la herramienta Backasting (Ver tabla 4).

\section{Tabla 4}

Herramienta utilizada para desarrollar la fase estrategias para la empresa

\begin{tabular}{|c|c|c|}
\hline Herramientas & Ventajas & Limitaciones \\
\hline $\begin{array}{l}\text { Backasting } \\
\text { Herramienta } \\
\text { ayuda a diseñar } \\
\text { el proceso que } \\
\text { se seguirá para } \\
\text { transitardel presente } \\
\text { al escenario apuesta }\end{array}$ & $\begin{array}{l}\checkmark \text { Se generan eventos principa- } \\
\text { les que deberán conducir al } \\
\text { logro del escenario apuesta } \\
\checkmark \text { Recuerda que el futuro no es } \\
\text { lineal y puede tener muchos } \\
\text { resultados alternativos de- } \\
\text { pendiendo de la toma de de- } \\
\text { cisiones } \\
\checkmark \text { Evita la extrapolación de las } \\
\text { condiciones actuales }\end{array}$ & $\begin{array}{l}\checkmark \text { Necesidad de actua- } \\
\text { lización constante } \\
\checkmark \text { Necesidad de contar } \\
\text { con recurso humano } \\
\text { capacitado }\end{array}$ \\
\hline
\end{tabular}

Fuente: Elaboración propia con información obtenida de Jackson (2013) para la realización del Backasting.

12 En la prospectiva estratégica el "escenario apuesta" se refiere al mejor escenario futuro seleccionado por el tomador de decisiones, el cual diseñará un conjunto de estrategias y acciones para construirla. 
Para la aplicación de la metodología prospectiva en una Mipyme es importante que ésta defina su objeto de estudio y el horizonte de tiempo que abarcará. Con relación al primero, es necesario que el propietario y/o gestor de la empresa no se enfoquen solo al giro de negocio para delimitar el objeto de estudio y, por el contrario, es importante que tengan una visión más holística del giro de negocio" ${ }^{13}$; así lo señala Ortega (2014: 43) "Si bien una empresa tiene claro el giro del negocio [...], ese no debe ser necesariamente el foco de atención del estudio, sino que es conveniente emplear una visión ampliada del giro, poniendo especial énfasis en sus variantes en el futuro".

Luego, con relación al horizonte temporal, la prospectiva determina que cada sector tiene su propio horizonte temporal, no obstante, para los estudios prospectivos se considera el corto plazo como un lapso de 5 años, el mediano un plazo de 5 a 10 años y el largo plazo después de los10 años. En el caso particular de las Mipymes se recomienda que el estudio prospectivo abarque el corto y mediano plazo, en principio, entre los 3 y 5 años, cuando la estructura y gestión de la Mipyme es limitada, y de 5 años a más, cuando la Mipyme está consolidada en el mercado y tiene grandes posibilidades de proyección.

En cuanto a la conformación del equipo de trabajo que llevará adelante el proceso de prospectiva e inteligencia empresarial, es necesario que en su formación se consideren a las áreas más importantes de la empresa: propietario(s), gestores, recursos humanos, administración, logística, producción, entre otras. Además, es necesario que se designe al líder o lideres responsables del proyecto, los cuales asumirán obligaciones y asignarán responsabilidades.

También, se recomienda que el grupo de trabajo sea multidisciplinario y esté compuesto por 2 ó 3 trabajadores cercanos al propietario y/o gestor de la empresa y que tengan una antigüedad de por lo menos 2 años en la empresa. Asimismo, es importante que al menos uno de los miembros que dirigirá el proceso tenga conocimientos de prospectiva e inteligencia empresarial, en caso contrario, se debe contratar a un consultor externo para que pueda acompañar en la aplicación

13 Por ejemplo, si una Mipyme se dedica a la fabricación de calzados de cuero, al realizar su estudio prospectivo no solo debería enfocarse en el futuro de los calzados de cuero, sino que debería ampliar su visión y estudiar la industria del calzado e identificar nuevas tecnologías, nuevos materiales para la fabricación de zapatos, etc. 
de la metodología y pueda fungir como capacitador para que en futuras ocasiones el grupo de trabajo pueda llevar adelante el estudio prospectivo.

Finalmente, el grupo de trabajo debe involucrar a través de un proceso participativo a las demás áreas de la organización (logística, ventas, administración, etc.), igualmente, se debe involucrar al propietario de la organización para generar mayor certeza en el proyecto.

\section{CONCLUSIONES}

La estructura empresarial y económica de un país está constituida casi en su totalidad por las Mipymes, éstas son valiosas para el desarrollo del país que las acoge; no obstante, su importancia no solo se circunscribe a aspectos económicos, pues, también, son generadoras de empleo, contribuyen al desarrollo productivo, aportan al Producto Bruto Interno (PBI), impulsan el desarrollo social, entre otros. Esto demuestra la necesidad de generar estrategias innovadoras para propiciar su consolidación y trascendencia en el tiempo.

Las Mipymes se encuentran inmersas en un entorno de constante e incesante competitividad e indeterminación. Consiguientemente, para hacer frente a dichas circunstancias necesitan desarrollar procesos de gestión y toma de decisiones anticipadas, con el objetivo de prever los cambios sociales y tecnológicos, identificar el ingreso de nuevos competidores y nuevos productos, anticiparse a los cambios en el mercado, conocer los actores y variables preponderantes, entre otros.

La prospectiva e inteligencia estratégica son técnicas innovadoras para la gestión y planeación de las Mipymes, puesto que, facilitan la toma de decisiones anticipadas desde una perspectiva sistémica y holística. En efecto, la metodología desarrollada hace uso de herramientas prospectivas y de inteligencia estratégica para la generación de estrategias de mediano y largo plazo, las cuales coadyuvarán a la construcción del futuro deseado de las Mipymes.

El presente artículo es el avance de una investigación más amplia que contempla aplicar la metodología propuesta a un conjunto de Mipymes, con el objetivo de validarla y generar información de corte cuantitativo y cualitativo relacionado al proceso de toma de decisiones y planeación a mediano y largo plazo. 


\section{REFERENCIAS}

(1) Aguirre, J. (2015). Inteligencia estratégica: un sistema para gestionar la innovación. Estudios Gerenciales. 31(134), pp.100-110.

(2) Bernal, C. (2010). Metodología de la investigación. Administración, economía, humanidades y ciencias sociales. $\left(3^{\circ} \mathrm{Ed}\right)$. Colombia: Pearson.

(3) Cabrera, L. (2015). La inteligencia estratégica: una herramienta necesaria para la toma de decisiones en el estado del siglo XXI. Revista de Política y Seguridad Pública. 2(5), pp.184-208.

(4) Correa, F., Leiva, V., y Stumpo, G. (2018). Mipymes y heterogeneidad estructural en América Latina. En Dini, M., y Stumpo, G. (Coord.). Mipymes en América Latina. Un frágil desempeño y nuevos desafios para las politicas de fomento. (pp. 473-543). [En línea] Disponible en: https://repositorio.cepal.org/bitstream/handle/11362/44148/1/S1800707_ es.pdf Recuperado: 18 de enero de 2019.

(5) Cubillo, J. (1997). La inteligencia empresarial en las pequeñas y medianas empresas competitivas de América Latina - algunas reflexiones. Brasília, 26(3), pp. 260-267.

(6) Dini, M., y Rueda, M. (2018). Avances y desafíos de las políticas de fomento de las mipymes. En Dini, M., y Stumpo, G. (Coord.). Mipymes en América Latina. Un frágil desempeño y nuevos desafios para las políticas de fomento. (pp. 9-34). [En línea] Disponible en: https://repositorio.cepal.org/bitstream/handle/11362/44148/1/S1800707_es.pdf Recuperado: 23 de enero de 2019.

(7) Dini, M., y Stumpo, G. (Coord.) (2018). Mipymes en América Latina. Un frágil desempeño y nuevos desafios para las políticas de fomento. [En línea] Disponible en: https://repositorio.cepal.org/bitstream/handle/11362/44148/1/S1800707_es.pdf Recuperado: 17 de enero de 2019.

(8) Gándara, G., Muñoz, A., y Luna, N. (2014). Árbol de competencias de Marc Giget. En Gándara, G., y Osorio, F. (Comp.) Métodos prospectivos: Manual para el estudio y la construcción del futuro. (pp. 97-119). México D.F, México: Paidós.

(9) Gándara, G., y Osorio, F. (Comp.). (2014) Métodos prospectivos: Manual para el estudio y la construcción del futuro. México D.F, México: Paidós. 
(10) Godet, M., y Durance, P. (2 Ed.) (2007). Prospectiva Estratégica: Problemas y métodos. [En línea] Disponible en: http://www.prospektiker.es/prospectiva/caja-herramientas-2007. pdf Recuperado: 18 de octubre de 2018.

(11) Goldstein, E., y Kulfas, M. (2011). Alcances y limitaciones de las políticas de apoyo a las pymes de América Latina. Debates para un nuevo marco conceptual y de implementación. En C. Ferraro. (Comp), Apoyando a las pymes: Políticas de fomento en América Latina y el Caribe. Santiago de Chile, Chile: Naciones Unidas-CEPAL. (pp. 429-507).

(12) Henriquez, L. (2009). Políticas para las Mipymes frente a la crisis. [En línea] Disponible en: http://www.ilo.org/wcmsp5/groups/public/---americas/---ro-lima/---sro-santiago/documents/publication/wcms_191351.pdf Recuperado: 22 de febrero de 2019.

(13) Intxaurburu, M. y Ochoa, C. (2005). Una revisión teórica de la herramienta de Benchmarking. Revista de Dirección y Administración de empresas. (12), pp. 73-103. [En línea] Disponible en: https:/www.ehu.eus/documents/2069587/2113623/12_6.pdf Recuperado: 23 de febrero de 2019.

(14) Jackson, M. (2013). Practical Foresight Guide. Chapter 3 - Methods. [En línea] Disponible en: https://www.shapingtomorrow.com/media-centre/pf-ch03.pdf Recuperado: 10 de febrero de 2019.

(15) Lacayo, M., y García, S. (s.f.). Principales problemas que impiden la permanencia y crecimiento de las pequeñas y medianas empresas mexicanas. [En línea] Disponible en: http:// www.aeca1.org/pub/on_line/comunicaciones_aal2011/cd/62c.pdf Recuperado: 19 de marzo de 2019.

(16) Medina, J. (s.f.). Prospectiva y empresa. [En línea] Disponible en: https://portal.concytec. gob.pe/images/noticias/jueves_congreso_innotec.pdf Recuperado: 10 de octubre de 2018.

(17) Ministerio de Planificación Nacional y Política Económica [Mideplan] (2015). La prospectiva y técnicas para elaborar escenarios a futuro. San José, Costa Rica: Mideplan. [En línea] Disponible en: http://www2.udec.cl/ rea/REVISTA\%20PDF/Rev70/art2.pdf Recuperado: 27 de marzo de 2019.

(18) Miklos, T., y Arroyo, M. (2011). Planeación y prospectiva estratégica. En Zeraroui, Z., y Balbi, E. (Coords.) Introducción a la prospectiva. (pp. 119-136). Puebla, México: Montiel y Soriano Editores. 
(19) Miklos, T., Jiménez. E., y Arroyo, M. (2010). Prospectiva, gobernabilidad y riesgo político: instrumentos para la acción. México, D.F., México: Limusa.

(20) Navarro, D. (2004). El ciclo de la inteligencia y sus límites. Cuadernos constitucionales de la Cátedra Fadrique Furió Ceriol. (48), pp. 51-66

(21) Organización Mundial del Comercio. (2016). Informe sobre el comercio mundial 2016. Igualdad de condiciones para el comercio de las pymes. [En línea] Disponible en: https://www.wto.org/spanish/res_s/booksp_s/world_trade_report16_s.pdf Recuperado: 14 de enero de 2019.

(22) Ortega, F. (2014). Prospectiva Empresarial. Manual de corporate foresight para América Latina. Lima, Perú: Fondo editorial de la Universidad de Lima.

(23) Shulsky, A., y Schmitt, G. (1992). Silent Warfare. Understanding the World (3ra ed). Washington, D.C., USA: Potomac Books.

(24) Serra del Pino, J., y Herrera, C. (marzo, 2017). Prospectiva y empresas: ¿cómo anticipar e influir en el futuro? [En línea] Disponible en: https://www.weforum.org/es/agenda/2017/03/prospectiva-y-empresas-como-anticipar-e-influir-en-el-futuro Recuperado: 12 de noviembre de 2019.

(25) Troy, T. (1992). The correct definition of intelligence. International Journal of Intelligence and Counterintelligence. 5(4), pp. 433-454. 